\title{
ESTUDIO DE LA BIOSORCIÓN DE Cd (II) Y Pb (II) USANDO COMO ADSORBENTE Nostoc sphaericum Vaucher
}

\author{
Roberto Carlos Chuquilín Goicochea ${ }^{* a}$, Dyana Daysi Rosales Laguna ${ }^{\mathrm{b}}$
}

\begin{abstract}
RESUMEN
Se evaluó la capacidad de biosorción del Nostoc sphaericum Vaucher en sistema por lotes. Las condiciones fueron: concentración de metal entre 40 y $240 \mathrm{ppm}, \mathrm{pH}$ de 2 a 5; 0,13 $\mathrm{g}$ de nostoc seco por $50 \mathrm{~mL}$ de solución, a $25{ }^{\circ} \mathrm{C}$ y en agitación. Las concentraciones de $\mathrm{Cd}$ (II) y $\mathrm{Pb}$ (II) se determinaron por Espectroscopía de Absorción Atómica. El modelo de isoterma de Langmuir se ajustó para $\mathrm{Pb}$ (II) $\left(\mathrm{R}^{2}=99,97 \%, \mathrm{p}>0,00\right)$, con $q_{\max }$ de $185,83 \mathrm{mg} / \mathrm{g}$ de Nostoc y Kd de 0,095 L/g, sin embargo, ninguno de los modelos estudiados explicaron los resultados para $\mathrm{Cd}$ (II). Se demostró que el $\mathrm{pH}$ es directamente proporcional a la capacidad de biosorción para $\mathrm{Pb}$ (II), siendo máxima a pH 5 ( $\mathrm{q}=37,43 \mathrm{mg} \mathrm{Cd} / \mathrm{g}$ de Nostoc). La biosorción de Cd (II) fue máxima a $\mathrm{pH} 3(\mathrm{q}=17,12 \mathrm{mg} \mathrm{Cd} / \mathrm{g}$ de Nostoc) sin mostrar comportamiento definido a $\mathrm{pH}$ mayores o menores a 3. La cinética de biosorción de $\mathrm{Cd}$ (II) y $\mathrm{Pb}$ (II) se ajustó a un modelo de pseudo segundo orden $\left(\mathrm{R}^{2}=98,38 \%\right.$ y $99,99 \%$, respectivamente, $\left.\mathrm{p}<0,05\right)$. El tamizaje fitoquímico demostró la presencia de saponinas, azúcares reductores y aminoácidos.
\end{abstract}

Palabras clave: Nostoc sphaericum, biosorción, isoterma, cinética, $\mathrm{Cd}, \mathrm{Pb}$.

\section{STUDY OF THE BIOSORPTION OF Cd (II) AND Pb (II) USING AS ADSORBENT Nostoc sphaericum Vaucher}

\author{
Roberto Carlos Chuquilín Goicochea ${ }^{* a}$, Dyana Daysi Rosales Laguna ${ }^{\mathrm{b}}$
}

\begin{abstract}
The biosorption capacity of the Nostoc sphaericum Vaucher in a batch system was evaluated. The conditions were: metal concentration between 40 and $240 \mathrm{ppm}, \mathrm{pH}$ of 2 to $5 ; 0,13 \mathrm{~g}$ of dried nostoc per $50 \mathrm{~mL}$ of solution, to $25^{\circ} \mathrm{C}$ and stirring. The concentrations of $\mathrm{Cd}$ (II) and $\mathrm{Pb}$ (II) were determined by Atomic Absorption Spectroscopy. The Langmuir model was adjusted for $\mathrm{Pb}$ (II) $\left(\mathrm{R}^{2}=99,97 \%, \mathrm{p}>0,00\right)$, with $q_{\max }$ of $185,83 \mathrm{mg} / \mathrm{g}$ of nostoc and $\mathrm{Kd}$ of 0,095 $\mathrm{L} / \mathrm{g}$; however, neither of the models studied explained the results for Cd (II). It was shown that the $\mathrm{pH}$ is directly proportional to the biosorption capacity for $\mathrm{Pb}$ (II), being maxim for $\mathrm{pH} 5(\mathrm{q}=37,43 \mathrm{mg} \mathrm{Cd} / \mathrm{g}$ of nostoc). The biosorption of $\mathrm{Cd}$ (II) was maxim to $\mathrm{pH} 3$ ( $\mathrm{q}=$
\end{abstract}

\footnotetext{
a Facultad de Ingeniería Química, Universidad Nacional del Centro del Perú. Dirección: Av. Mariscal Castilla, 3909, Huancayo. robercar124@hotmail.com

${ }^{\mathrm{b}}$ Facultad de Ciencias Agrarias, Universidad Nacional Hermilio Valdizán, Huánuco.
} 
$17,12 \mathrm{mg} \mathrm{Cd} / \mathrm{g}$ of nostoc) without showing behavior defined $\mathrm{pH}$ of higher or lower than 3. The adsorption kinetics of $\mathrm{Cd}$ (II) and $\mathrm{Pb}$ (II) to a pseudo second order model was fitted ( $\mathrm{R} 2=98,38 \%$ and $99,99 \%$ respectively, $\mathrm{p}<0,05$ ). The phytochemical screening showed the presence of saponins, reducer sugars and amino acids.

Key words: Nostoc sphaericum, biosorption, isotherm, kinetics, $\mathrm{Cd}, \mathrm{Pb}$.

\section{INTRODUCCIÓN}

Nostoc sphaericum Vaucher es el nombre científico con el que se conoce al popular "Cushuro", voz quechua que significa "crespo" no sólo por el aspecto, color y consistencia, sino por su misteriosa aparición tras la lluvia, como salido de la nada. Forma al principio colonias esféricas que luego se aplanan, de textura membranosa, coriácea, de color verde oliva o pardo verdoso o amarillento, envuelta por una capa externa firme que puede alcanzar varios centímetros. Dichas colonias generan biodermas algales que, cuando están secos, se asemejan a delgados y transparentes papeles negros. El polisacárido extracelular de estos organismos es un complejo de alto peso molecular con propiedades reológicas ${ }^{1}$.

El cromatograma en papel descendente reveló que el polisacárido está constituido por unidades urónicas y que además encontramos acompañándolo azúcares como galactosa, manosa, xilosa y ramnosa. El espectro infrarrojo confirma lo anterior ya que presenta picos correspondientes a grupos carboxilos e hidroxilos ${ }^{2}$.

La biosorción como proceso fisicoquímico consiste en la retención en un sólido adsorbente de origen biológico, llamado biosorbente, de especies químicas contaminantes llamadas adsorbatos o solutos contenidos inicialmente en una fase líquida acuosa, son importantes para ello la química en medio acuoso del soluto y del biosorbente, sus grupos funcionales, la solubilidad, tamaño y porosidad de la partícula. Si se mantiene en contacto a ambos en determinadas condiciones de concentración, $\mathrm{pH}$, temperatura, agitación y tiempo, se desarrollará un proceso que puede ser descrito por parámetros y modelos matemáticos adecuados ${ }^{3}$. Los metales pesados bajo su forma iónica son vertidos a ríos y mares provenientes de industrias como de curtiembre, fotográfica, pigmentos, plásticos, de baterías y metalúrgica sin el debido control ambiental ${ }^{4}$.

La concentración de la biomasa es una variable que puede afectar la biosorción del metal ${ }^{5}$. Los datos revisados sugieren que este efecto de disminución de la capacidad de adsorción conforme aumenta la concentración celular puede deberse a que ocurren interacciones electrostáticas entre las células, lo cual podría causar una disminución en los sitios de unión de la pared celular por la aglomeración de éstas, es decir, conforme la distancia es mayor, se adsorbe una mayor cantidad de cationes ${ }^{5}$.

El pH de la solución tiene un efecto significativo en la biosorción del metal ya que lo influencian tanto los sitios de unión de la superficie de la célula como las características 
químicas de la solución. El comportamiento general observado para diferentes tipos de metales y biosorbentes, es que la biosorción del metal es despreciable a valores de $\mathrm{pH}$ entre 1 y 2 y se incrementa conforme el $\mathrm{pH}$ aumenta hasta alcanzar un máximo a valores entre 5 y $7^{5}$. La dependencia del $\mathrm{pH}$ también fue mostrada cuando se utilizó biomasa inmovilizada obteniéndose biosorción máxima a valores de $\mathrm{pH} 6$ y 7 , con reducciones en la capacidad de biosorción hasta de un $50 \%$ cuando el $\mathrm{pH}$ fue de $2^{6}$.

En general, los autores anteriormente citados sugieren que a bajos valores de $\mathrm{pH}$ en donde existe una alta concentración de protones, los cationes metálicos y los protones compiten por los sitios de unión de la pared celular, lo cual da como resultado que debido a fuerzas de repulsión, la captura del metal disminuya; conforme el $\mathrm{pH}$ se incrementa, se exponen más sitios de unión con carga negativa provocando que la atracción por iones positivamente cargados se incremente.

El modelo de Elovich es útil para determinar la cinética de quimiosorción de gases sobre sólidos, pero también se utiliza para describir la adsorción de contaminantes en soluciones acuosas $^{7,8}$. El modelo de pseudo segundo orden se utiliza para determinar la cinética de quimiosorción dada por la formación de enlaces químicos (interacciones fuertes) entre el adsorbente y el adsorbato, en una monocapa superficial ${ }^{9,10}$.

\section{PARTE EXPERIMENTAL}

\section{Colecta y transporte del material biosorbente}

Se obtuvo "cushuro" de la laguna de Nahuimpuquio, provincia de Huancayo, en una masa de $10 \mathrm{~kg}$. Se secó mediante aire caliente a $65^{\circ} \mathrm{C}$ por $24 \mathrm{~h}$, hasta que se alcance una masa seca con $3 \%$ de humedad. Luego se redujo el tamaño de partícula con un molino de cizalla y se envasó en bolsas de polietileno de $1 / 2 \mathrm{~kg}$, para luego ser almacenado hasta su uso.

\section{Acondicionamiento de las soluciones contaminadas}

Se prepararon soluciones acuosas de sulfato de cadmio y nitrato de plomo, para simular agua contaminada con $\mathrm{Cd}$ (II) y $\mathrm{Pb}$ (II), respectivamente. Estas soluciones de concentración de $100 \mathrm{ppm}$ se mantuvieron a $\mathrm{pH} 5$ y temperatura de $25^{\circ} \mathrm{C}$.

\section{Proceso de biosorción en un sistema por lotes}

Se disolvieron $0,13 \mathrm{~g}$ de Nostoc deshidratado en vasos de precipitación de $100 \mathrm{~mL}$ con 50 $\mathrm{mL}$ de soluciones contaminadas con los metales pesados en estudio. Se mantuvo en agitación durante 6 horas hasta alcanzar en equilibrio (hasta que no haya adsorción y/o desorción).

\section{Determinación de la cantidad de metal pesado}

Se utilizó la técnica de Espectroscopía de Absorción Atómica, para analizar el agua después del proceso de biosorción, se usó un espectrofotómetro de absorción atómica equipado por flama modelo AA Analyst 300. 


\section{Estimación de las isotermas de adsorción}

Se estimaron tres isotermas de adsorción, que son las más usadas en estos casos: Lineal, Freundlich y Langmuir ${ }^{11}$.

\section{Estimación de la cinética de biosorción}

Para analizar la cinética de adsorción de Cd (II) y $\mathrm{Pb}$ (II), se estimaron dos modelos de la cinética de adsorción: Elovich y pseudo segundo orden.

\section{Análisis estadístico de los datos}

Se realizó el análisis de regresión lineal es una técnica estadística utilizada para estudiar la relación entre variables. Se adapta a una amplia variedad de situaciones. El análisis de varianza de la regresión ayudó a revelar si una ecuación de regresión lineal simple es significativa. El análisis estadístico se realizó con el MS Excel y Minitab versión 16.

\section{RESULTADOS Y DISCUSIÓN}

\section{Resultados de biosorción de Cd (II)}

\section{a) Evaluación de modelos de isotermas de biosorción}

En la figura 1 se observa que, la isoterma lineal presentó una constante de equilibrio $\mathrm{K}$ es 0,085 L de solución por g de Nostoc, pero el $\mathrm{R}^{2}$ fue $61,87 \%$ que aunque disminuyó, fue superior al de las demás isotermas evaluadas. La isoterma de Freundlich tuvo un valor $\mathrm{n}$ de 0,297; se dice que, cuando las isotermas son cóncavas hacia el eje de las abscisas, $\mathrm{n}<1$ la isoterma de Freundlich es favorable ${ }^{11}$; así mismo, la constante de afinidad (K) fue de 3.98 L de solución por g de Nostoc; sin embargo, el $\mathrm{R}^{2}$ fue $54,78 \%$ lo que indica una bajísima correlación. El modelo de Langmuir, tuvo una capacidad biosortiva específica máxima de Cd (II) $\left(q_{\max }\right)$ de 13,93 mg de Cd (II) por g de Nostoc, y la constante de disociación $\left(\mathrm{K}_{\mathrm{d}}\right)$ fue $6,19 \mathrm{mg}$ de $\mathrm{Cd}$ (II) por L solución; el coeficiente $\mathrm{R}^{2}$ fue $45,15 \%$ y al igual que en los otros casos el valor fue bajo.

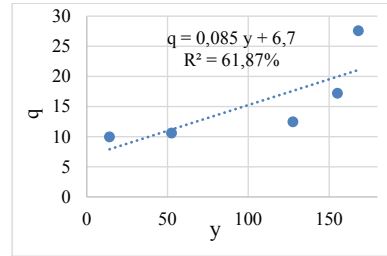

a)

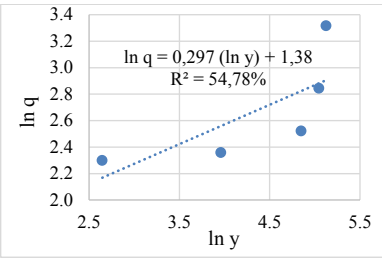

b)

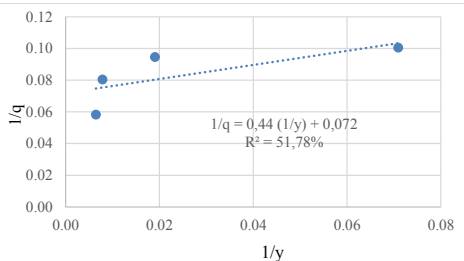

c)

Figura 1. Modelo de isoterma de biosorción de Cd (II): a) Lineal, b) Freundlich y c) Langmuir 
Se observa que, en el análisis de varianza ningún modelo de isoterma se ajusta a los datos de Cd (II) $(\mathrm{p}>0,05)$.

Tabla 1. Análisis de varianza de modelos de isotermas de biosorción de Cd (II).

\begin{tabular}{llcc}
\hline \multicolumn{1}{c}{ Isoterma } & \multicolumn{1}{c}{ Ecuación } & $\mathbf{R}^{2}$ & Valor $\mathbf{p}$ \\
\hline Lineal & $\mathrm{q}=0,085 \mathrm{y}+6,7$ & $61,87 \%$ & 0,11 \\
Freundlich & $\ln \mathrm{q}=0,297 \ln \mathrm{y}+1,38$ & $54,78 \%$ & 0,15 \\
Langmuir & $1 / \mathrm{q}=0,641 / \mathrm{y}+0,06$ & $45,10 \%$ & 0,21 \\
\hline
\end{tabular}

* Significativo a un nivel de significancia de 0.05 .

\section{b) Efecto del pH en la biosorción de Cd (II)}

Se usaron cuatro valores de $\mathrm{pH}: 2,3,4$ y 5; pero a un valor de 3 , se presentó una alta capacidad biosortiva específica de Cd (II) (17,12 mg de Cd (II) por g de Nostoc), lo que equivale a $44,51 \%$ de remoción de $\mathrm{Cd}$ (II). Además a $\mathrm{pH}$ menor o mayor a 3 no hubo un comportamiento o tendencia definida.

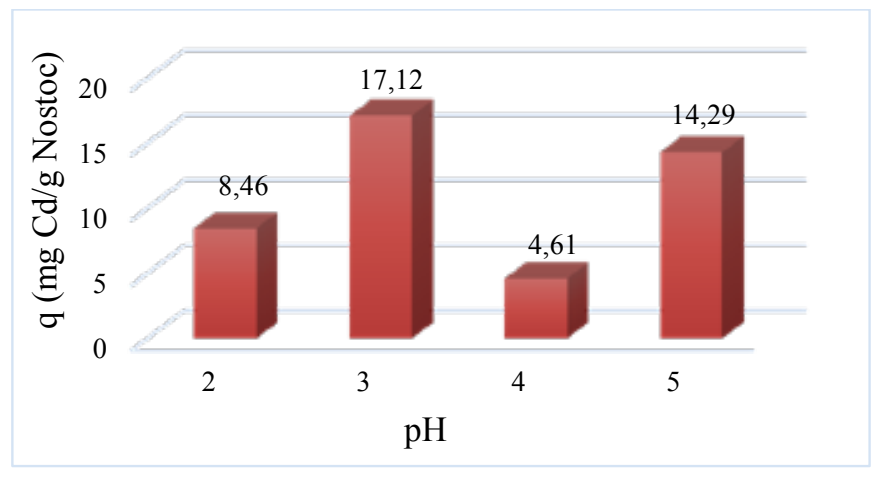

Figura 2. Efecto del pH sobre la biosorción de Cd (II).

\section{c) Cinética de biosorción de Cd (II)}

Se evaluaron el modelo de Elovich, que es utilizado para describir la cinética de adsorción de sistemas inorgánicos como es el caso del Cd (II), y el modelo de pseudo segundo orden, que representa la quimiosorción o adsorción química debido a la formación de enlaces químicos (interacciones fuertes) entre el adsorbente y el adsorbato en una monocapa en la superficie $^{12}$. En la figura 3, la ecuación de Elovich tuvo un $\mathrm{R}^{2}$ de 70,91\%; los resultados de la pendiente y la intersección con la ordenada se utilizaron para determinar los valores de las constantes $\alpha$ (9,03 $\mathrm{mg}$ de Cd (II) por g de Nostoc) y $\beta$ (1,85 mg de Cd (II) por g de Nostoc), con los cuales se calculó la constante en equilibrio $\mathrm{K}_{\mathrm{e}}=\alpha / \beta=4,89$ (adimensional). En el modelo de pseudo segundo orden, tuvo un $\mathrm{R}^{2}$ de $98,38 \%$; se observa que, la capacidad biosortiva específica de Cd (II) en equilibrio $\left(\mathrm{q}_{\mathrm{e}}\right)$ fue $18,97 \mathrm{mg} \mathrm{Cd}$ (II) por g de Nostoc, y la constante $\mathrm{K}_{2}^{\prime}$ de unidades $\mathrm{mg}$ de $\mathrm{Cd}$ (II) por $\mathrm{g}$ de Nostoc por minuto fue $0,007 \mathrm{mg} / \mathrm{g}$.min. 


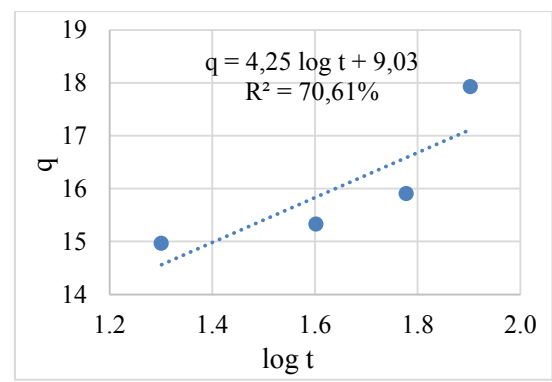

a)

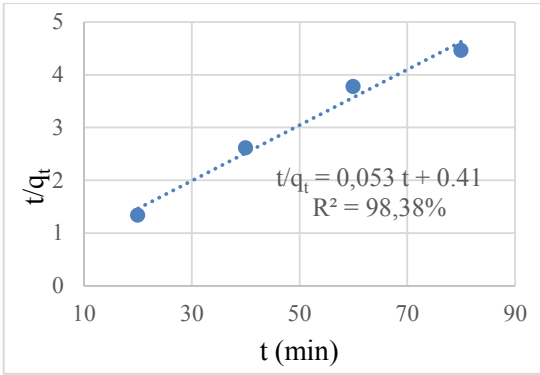

b)

Figura 3. Modelos cinéticos de biosorción de Cd (II): a) de Elovich y b) Pseudo segundo orden.

La tabla 2 muestra que, la cinética de biosorción de Cd (II) sigue un modelo matemático de pseudo segundo orden, ya que resultó significativo $(p<0,05)$ y tuvo un alto $\mathrm{R}^{2}$. Este modelo ha sido reportado como dominante en los procesos de adsorción de metales, obteniendo buen ajuste $\left(\mathrm{R}^{2}=99 \%\right)$ para $\mathrm{Cd}$ (II) utilizando resinas ${ }^{12}$. La reacción de segundo orden indica que entran dos reactantes para dar un producto ${ }^{13}$, lo que probablemente ocurre con el Nostoc como adsorbente y el $\mathrm{Cd}$ (II) como adsorbato, que se unen para dar un producto en donde la superficie del Nostoc contendría Cd (II) biosorbido.

Tabla 2. Análisis de varianza de modelos cinéticos de biosorción de Cd (II).

\begin{tabular}{cccc}
\hline Modelo & Ecuación & $\mathbf{R}^{2}$ & Valor $\mathbf{p}$ \\
\hline Elovich & $\mathrm{q}_{\mathrm{t}}=9,03+4,25 \log \mathrm{t}$ & $70,61 \%$ & 0,16 \\
Pseudo segundo orden & $\mathrm{t} / \mathrm{q}_{\mathrm{t}}=0,41+0,053 \mathrm{t}$ & $98,38 \%$ & $0,008^{*}$ \\
\hline
\end{tabular}

* Significativo a un nivel de significancia de 0,05

\section{Resultados de biosorción de Pb (II)}

\section{a) Evaluación de modelos de isotermas de biosorción}

En la figura 4 se muestra que, la isoterma lineal tuvo una constante de equilibrio $\mathrm{K}$ es $2,74 \mathrm{~L}$ de solución por $\mathrm{g}$ de Nostoc y un $\mathrm{R}^{2}$ de $79,52 \%$ que fue regular. En la isoterma de Freundlich, el valor de " $n$ " 0,65 es favorable ${ }^{11}$, el $\mathrm{R}^{2}$ fue $90,8 \%$ y la constante de afinidad " $\mathrm{K}$ " fue de 13,33 L de solución por $\mathrm{g}$ de Nostoc. La isoterma de Langmuir, tuvo un $\mathrm{R}^{2}$ $=99,13 \%$, un valor $\mathrm{K}_{\mathrm{d}}$ de 17,66 mg de $\mathrm{Pb}$ (II) por L de solución y capacidad biosortiva específica máxima $\left(\mathrm{q}_{\max }\right)$ fue $185,9 \mathrm{mg}$ de $\mathrm{Pb}$ (II) por $\mathrm{g}$ de Nostoc. 


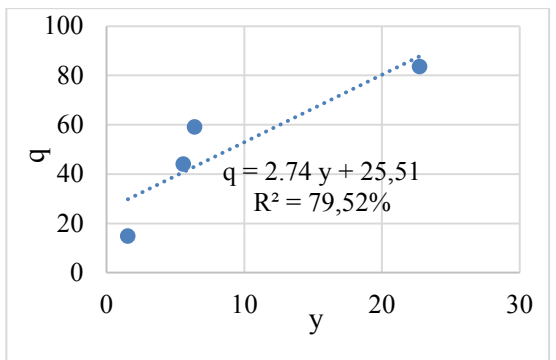

a)

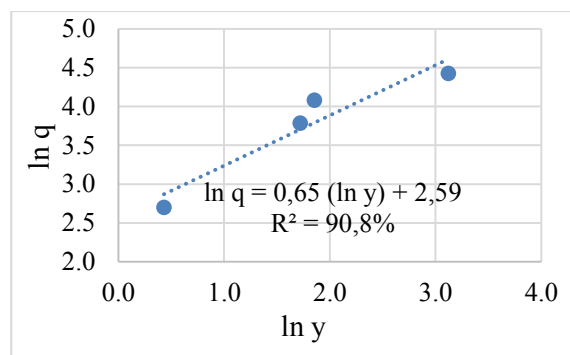

b)

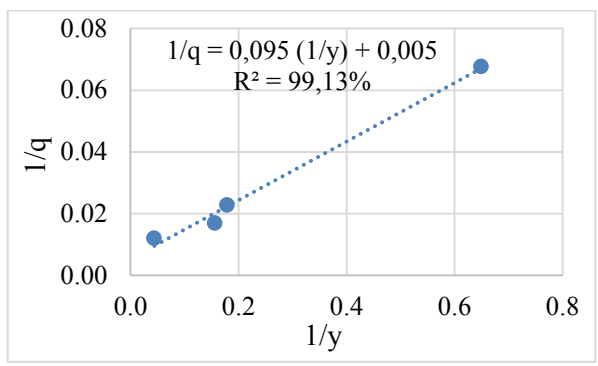

c)

Figura 4. Modelos de isotermas de biosorción de $\mathrm{Pb}$ (II): a) Lineal, b) Freundlich y c) Langmuir.

En la tabla 3, se observa que el modelo de Langmuir resultó significativo $(p<0,05)$, y tuvo el mayor $\mathrm{R}^{2}$ que fue $99,13 \%$. Por lo tanto, fue el modelo que mejor se ajustó a los datos de biosorción de $\mathrm{Pb}$ (II). Teóricamente la isoterma de Langmuir es un modelo que representa una interacción soluto - adsorbente de segundo orden y puede expresarse de la siguiente forma: Toda la superficie tiene la misma actividad para la adsorción; no hay interacción entre las especies adsorbidas; toda la adsorción tiene lugar mediante el mismo mecanismo y el grado de adsorción es inferior a una capa mono molecular completa en la superficie ${ }^{11}$.

Tabla 3. Análisis de varianza de modelos de isotermas de biosorción de $\mathrm{Pb}$ (II).

\begin{tabular}{llcc}
\hline \multicolumn{1}{c}{ Isoterma } & \multicolumn{1}{c}{ Ecuación } & $\mathbf{R}^{2}$ & Valor p \\
\hline Lineal & $\mathrm{y}=2,74 \mathrm{q}+25,51$ & $79,52 \%$ & 0,108 \\
Freundlich & $\ln \mathrm{y}=0,65 \ln \mathrm{q}+2,5902$ & $90,80 \%$ & $0,047 *$ \\
Langmuir & $1 / \mathrm{y}=0,0951 / \mathrm{q}+0,005$ & $99,13 \%$ & $0,004 *$ \\
\hline
\end{tabular}

* Significativo a un nivel de significancia de 0,05 . 


\section{b) Efecto del pH sobre la biosorción de Pb (II)}

En la figura 5 se observa que, el $\mathrm{pH}$ es directamente proporcional a la capacidad biosortiva específica de $\mathrm{Pb}$ (II), llegando a alcanzar el máximo valor de " $\mathrm{q}$ ” a $\mathrm{pH} 5,0$, siendo del orden de 37,43 mg de $\mathrm{Pb}$ (II) por g de Nostoc, lo que equivale a una remoción del 97,32\% del $\mathrm{Pb}$ (II).

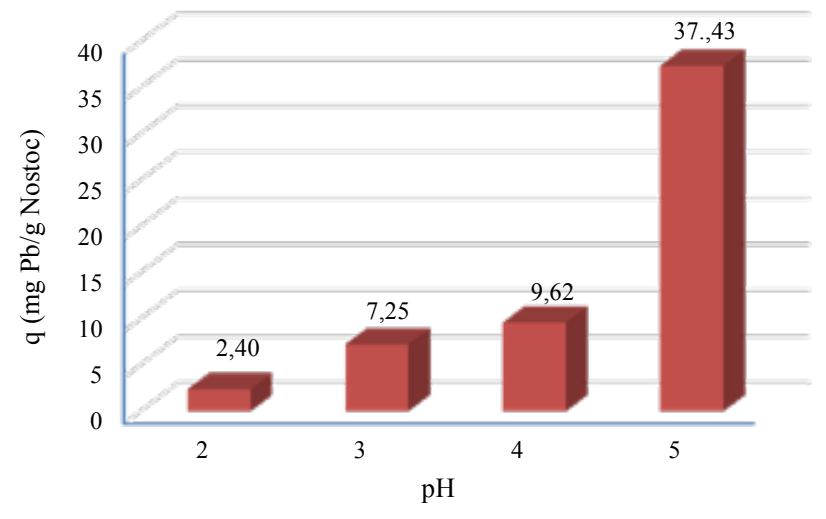

Figura 5. Efecto del pH sobre la biosorción de $\mathrm{Pb}$ (II).

\section{c) Cinética de biosorción de $\mathrm{Pb}$ (II)}

El modelo de Elovich mostró un coeficiente de determinación $\left(\mathrm{R}^{2}\right)$ de $70,61 \%$. Los resultados de la pendiente y ordenada al origen se utilizaron para determinar los valores de las constantes $\alpha$ y $\beta$, con los cuales se calculó la constante en equilibrio $\mathrm{Ke}=\alpha / \beta$. Siendo la constante de equilibrio $\mathrm{Ke}=50$ (adimensional). En el modelo cinético pseudo segundo orden, el coeficiente de determinación $\left(\mathrm{R}^{2}\right)$ fue de 99,99\%. Los modelos de cinética de pseudo segundo orden han sido reportados con buen ajuste de coeficiente $\mathrm{R}^{2}=99 \%$ para $\mathrm{Pb}$ (II) utilizando resinas ${ }^{12}$ (figura 6).

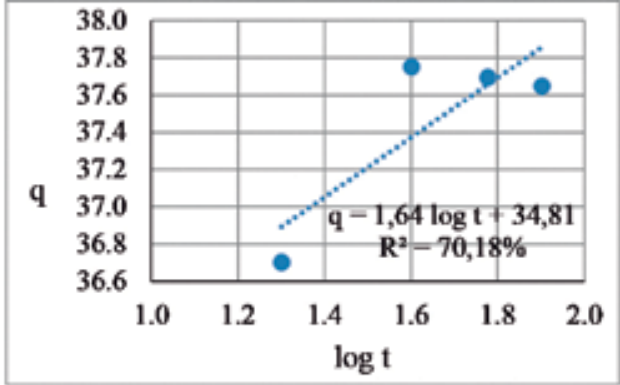

a)

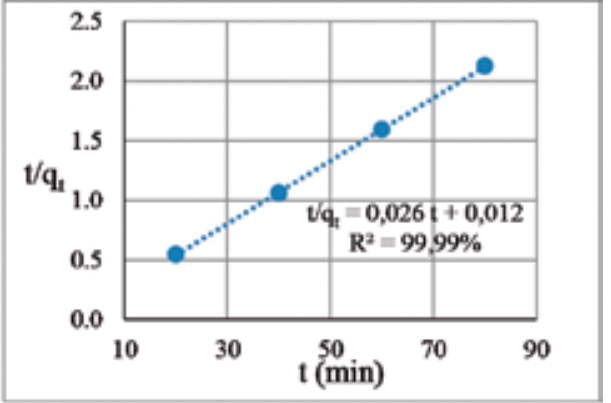

b)

Figura 6. Modelos cinéticos de biosorción de $\mathrm{Pb}$ (II): a) de Elovich y b) Pseudo segundo orden. 
Los valores en la tabla 4 muestran que la biosorción de $\mathrm{Pb}$ (II) sigue un modelo de pseudo segundo orden, ya que resultó significativo $(p<0,05)$ y tuvo un alto $\mathrm{R}^{2}$. Esta evidencia equivale a decir que, el proceso de biosorción de $\mathrm{Pb}$ (II) es una quimiosorción ${ }^{14}$. Estos resultados de cinética coinciden con la isoterma Langmuir, que representa a una reacción de segundo orden, es decir, entran en reacción el Nostoc deshidratado como adsorbente y el $\mathrm{Pb}$ (II) como adsorbato, que se unen para dar como producto el Nostoc con su superficie probablemente llena de $\mathrm{Pb}$ (II) adsorbido ${ }^{13}$.

Tabla 4. Análisis de varianza de modelos cinéticos de biosorción de $\mathrm{Pb}$ (II).

\begin{tabular}{cccc}
\hline Modelo & \multicolumn{1}{c}{ Ecuación } & $\mathbf{R}^{2}$ & Valor p \\
\hline Elovich & $\mathrm{y}=1,604 \mathrm{q}_{\mathrm{t}}+34,81$ & $70,18 \%$ & 0,162 \\
Pseudo segundo orden & $\mathrm{t} / \mathrm{q}_{\mathrm{t}}=0.012+0,026 \mathrm{t}$ & $99,99 \%$ & $0,000 *$ \\
\hline
\end{tabular}

*Si* Significativo a un nivel de significancia de 0,05 .

\section{d) Caracterización del Nostoc sphaericum Vaucher Tamizaje fitoquímico}

El Nostoc sphaericum Vaucher dio positivo a la presencia de saponinas, azúcares reductores y aminoácidos, que poseen grupos carboxilos e hidroxilos. De acuerdo al trabajo realizado por Melgarejo $^{2}$, el cromatograma en papel descendente reveló que, el polisacárido del Nostoc sphaericum Vaucher está constituido por unidades urónicas y que además se encuentran acompañándolo azúcares como galactosa, manosa, xilosa y ramnosa. Agrega el autor que, el espectro infrarrojo confirma la presencia de picos correspondientes a grupos carboxilos e hidroxilos ${ }^{2}$. Estos grupos, explican con su presencia el poder adsorbente del Nostoc; ya que se ha evidenciado interacciones entre estos grupos funcionales y metales divalentes en diversos estudios ${ }^{2,6,15}$. En la tabla 5 se presentan los resultados de tamizaje fitoquímico. 
Tabla 5. Tamizaje fitoquímico del liofilizado de Nostoc sphaericum Vaucher.

\begin{tabular}{|c|c|c|c|}
\hline ENSAYOS & METABOLITOS & MUESTRA & RESULTADO \\
\hline \multicolumn{4}{|c|}{ Extracto diclorometano } \\
\hline $\begin{array}{c}\text { Liebermann- } \\
\text { Burchard }\end{array}$ & Triterpenos/esteroles & Droga & - \\
\hline Bornträger & Quinonas & Droga & - \\
\hline \multicolumn{4}{|c|}{ Extracto metanólico } \\
\hline $\begin{array}{c}\text { Liebermann- } \\
\text { Burchard }\end{array}$ & Triterpenos/esteroles & Droga & - \\
\hline Bornträger & Quinonas & Droga & - \\
\hline Shinoda & Flavonoides & Droga & - \\
\hline Cloruro férrico & Polifenoles & Droga & - \\
\hline Espuma & Saponinas & Droga & + \\
\hline Dragendorf & Alcaloides & Droga & - \\
\hline Mayer & Alcaloides & Droga & - \\
\hline Wagner & Alcaloides & Droga & - \\
\hline \multicolumn{4}{|c|}{ Extracto acuoso ácido } \\
\hline Dragendorf & Alcaloides & Droga & - \\
\hline Mayer & Alcaloides & Droga & - \\
\hline Wagner & Alcaloides & Droga & - \\
\hline \multicolumn{4}{|c|}{ Extracto acuoso } \\
\hline Shinoda & Flavonoides & Droga & - \\
\hline Espuma & Saponinas & Droga & + \\
\hline Fehling & Azúcares reductores & Droga & + \\
\hline Ninhidrina & Aminoácidos & Droga & + \\
\hline Cloruro férrico & Polifenoles & Droga & - \\
\hline Gelatina & Taninos & Droga & - \\
\hline
\end{tabular}

Leyenda: $(+)=$ Positivo $(-)=$ Negativo 


\section{CONCLUSIONES}

El modelo de isoterma de Langmuir resultó significativo $(\mathrm{p}<0,05)$ para describir la biosorción de $\mathrm{Pb}$ (II). La capacidad biosortiva específica máxima de $\mathrm{Pb}$ (II) $\left(\mathrm{q}_{\max }\right)$ fue de 185,89 mg/g y la constante de disociación $\left(\mathrm{K}_{\mathrm{d}}\right)$ es de $0,095 \mathrm{~L} / \mathrm{g}$; sin embargo, ningún modelo de isoterma estudiado resultó significativo $(\mathrm{p}>0,05)$ para describir la biosorción de $\mathrm{Cd}$ (II), en las condiciones estudiadas. A pH 3 la capacidad biosortiva específica de $\mathrm{Cd}$ (II) fue mayor ( $\mathrm{q}=$ $17,12 \mathrm{mg} / \mathrm{g}$ ), lo que equivale a remover el $44.51 \%$ de Cd (II), a pH mayores o menores a 3 la capacidad biosortiva específica no mostró un comportamiento definido; sin embargo, existe una relación directa y proporcional entre el $\mathrm{pH}$ y la capacidad biosortiva específica de $\mathrm{Pb}$ (II), siendo máxima a pH 5 (q=37,43 mg/g), lo que equivale a remover el 97,32\% de $\mathrm{Pb}$ (II). El modelo de pseudo segundo orden fue el que mejor describió la cinética de biosorción de $\mathrm{Cd}$ (II) y $\mathrm{Pb}$ (II) ( $\mathrm{R}^{2}=98,38 \%$ y 99,99\%, respectivamente), en un rango de 20 a $80 \mathrm{~min}$. El tamizaje fitoquímico reveló la presencia de saponinas, azúcares reductores y aminoácidos, los cuales presentan grupos carboxilos e hidroxilos, que serían responsables de la biosorción de Cd (II) y Pb (II).

\section{AGRADECIMIENTO}

A la Universidad Nacional del Centro del Perú, por el apoyo durante mi formación en posgrado y un especial agradecimiento al M.Sc. Luis Fernando Riccio Yauri por su asesoramiento. Al Dr. Segundo Guillermo Ruíz Reyes, Profesor Principal de la Facultad de Farmacia y Bioquímica de la Universidad Nacional de Trujillo, por su colaboración con el tamizaje fitoquímico del Nostoc.

\section{REFERENCIAS BIBLIOGRÁFICAS}

1. Campos D. Caracterización fisicoquímica del espejo de agua de la laguna Cushuro de la Provincia de Sánchez Carrión donde se desarrolla el Nostoc commune. Trujillo: Universidad Nacional de Trujillo; 2010.

2. Melgarejo H. Estudio químico bromatológico de Nostoc sphaericum Vaucher "Cushuro" procedente de la provincia de Concepción (Junín). Lima: Universidad Nacional Mayor de San Marcos; 1995.

3. Cuizano N, Reyes U, Domínguez S, Llanos B, Navarro E. Relevancia del pH en la adsorción de iones metálicos mediante algas pardas. Rev. de la Soc. Quím. del Perú. 2010; 76 (2): 123-130.

4. Volesky B. Removal and recovery of heavy metals by biosorption. Boca Ratón: CRC Press; 1990.

5. Modak J, Natarajan K. Biosorption of metals using nonliving biomass- A review. J Miner Metall Proc. 1995; 12(1): 89-96.

6. Luef E, Prey T, Kubiceck P. Biosorption of zinc by fungal mycelial waste. Appl Microbiol Biotechnol. 1991; 34(1): 688-692. 
7. Igwe J, Abia A. A bioseparation process for removing heavy metals from waste water using biosorbents. African J of Biotech. 2006; 5(12): 1167-1179.

8. Goswami S, Uday C. Studies of adsorption behaviour of Cr (VI) onto synthetichydrous stannic oxide. J Water SA. 2005; 31(4): 597-602.

9. Ho YS. Review of second-order models for adsorption systems. J Hazard Mater. 2006;136(3):681-689.

10. Paul S, Bera P, Chattioadhyay P, Ray L. Biosorption of Pb (II) by Bacillus cereus M116 inmovilized in calcium alginate gel. J Haz Subs Res. 2006; 5(2): 1-12.

11. Sánchez E, Garza T, Almaguer V, Saénz I, Liñán A. Estudio cinético e isotermas de adsorción de Ni (II) y Zn (II) utilizando la biomasa del alga Chlorella sp. Inmovilizada. Ciencia UANL. 2008; 11(2):168-176.

12. Paliulis D. Numerical modeling kinetics of heavy metal sorption from polluted water. $\mathbf{J}$ Envirom Eng Land Man. 2006; 14(1): 10-15.

13. Avery A.Cinética química básica y mecanismos de reacción. Barcelona: Editorial Reverté SA; 2002.

14. Wankasi $\mathrm{M}$. Sorption of $\mathrm{Pb}+2$ and $\mathrm{Cu}+2$ ions from aqueous solutions by chloride complexation. J Soil \& Sedim Contam. 2006; 11(6): 841-859.

15. Cuizano N, Navarro A. Biosorción de metales pesados por algas marinas: posible solución a la contaminación a bajas concentraciones. An Quím. 2008; 104(2): 120- 125. 